\title{
Panorama del nowcasting en los países de nuestro entorno
}

https://doi.org/10.31978/639-19-010-0.005

\author{
Luis María Bañón Peregrín'1 (LBanonP@aemet.es)
}

${ }^{1}$ AEMET / Delegación Territorial en la Región de Murcia

\begin{abstract}
RESUMEN
Este artículo explora diversos aspectos del nowcasting meteorológico, empezando por remarcar sus diferencias con la predicción a muy corto plazo. Repasa algunas de las necesidades de sus usuarios ya que la rápida caducidad de este tipo de predicciones exige productos muy adaptados. Por su parte, los sistemas de nowcasting han mejorado sus componentes. Se muestran algunos de estos avances en la observación meteorológica, en los análisis y en modelos numéricos de predicción del tiempo. Se destaca la tendencia hacia herramientas probabilísticas, y sin costuras, desde la observación hasta las primeras horas de predicción. Se presentan algunos ejemplos de sistemas operativos de nowcasting en los Servicios Meteorológicos Nacionales de nuestro entorno. Finalmente, se destaca el papel del predictor meteorológico en sus tareas de nowcasting, así como los problemas a los que se enfrenta.
\end{abstract}

PALABRAS CLAVE: nowcasting; predicción inmediata; predicción sin costuras; seamless prediction; blending; nowcaster.

\section{NOWCASTING O PREDICCIÓN A MUY CORTO PLAZO}

El término nowcasting comenzó con la observación meteorológica intensiva mediante teledetección, y tuvo su primer gran impulso a finales de la década de 1970, a raíz de unas campañas de predicción del tiempo de las primeras horas, que se basaron principalmente en el uso de imágenes de satélites geoestacionarios. A principios de los 80, se avanzó en su descripción, considerándose el nowcasting como la predicción meteorológica local enfocada en la observación intensiva con pronta disponibilidad, y en la que los sensores remotos jugaban un papel fundamental. (BROwNING, 1982).

Durante décadas, el término anglosajón nowcasting, traducido en ocasiones como «predicción inmediata», se consideró como la pura extrapolación lineal de los fenómenos meteorológicos existentes en un momento dado, hasta un periodo no más allá de las tres horas. Esta definición no consideraba al nowcasting como una verdadera predicción, tan solo una extrapolación de los fenómenos ya existentes. La verdadera predicción debería anticiparse a los nuevos desarrollos o fenómenos antes de que se produjeran. (INM, 198?).

En 2010, el grupo de investigación del nowcasting de la OMM definió el nowcasting como la predicción con detalle local, por cualquier método, desde el presente hasta las primeras 6 horas, incluyendo una descripción detallada del tiempo actual.

Por otro lado, la predicción meteorológica a muy corto plazo abarca las primeras 12 horas, incluyendo el periodo de predicción del nowcasting. Sin embargo, de este último se espera mayor detalle espacial y temporal, y una alta frecuencia de actualización, siendo menores tales exigencias en la predicción a muy corto plazo. 
Además, en el nowcasting, salvo excepciones, el papel del predictor es determinante, cediéndole responsabilidad a la automatización a medida que aumenta el rango de predicción. En general, el nowcasting enfoca su atención en fenómenos desde la microescala (hasta 2 km; ORLANSKI, 1975) a mesogamma (de 2 a 20 km), mientras que el muy corto plazo se enfoca a fenómenos algo mayores, de escalas mesogamma a mesoalfa (de 200 a 2000 km), incluso en las primeras horas de predicción. Aunque el nowcasting está abordando cada vez más variables y situaciones meteorológicas, su principal motivación ha sido la precipitación y las situaciones meteorológicas de alto impacto para los usuarios, y orientado a ubicaciones específicas (BrownING, 1982, y AмвRosetтi et al., 2014). La predicción a muy corto plazo abarca todo tipo de variables y situaciones, y su cobertura es más general en el espacio. Respecto a su utilidad, mientras que con esta última se acotan probabilidades ante posibles fenómenos y se preparan las medidas y acciones, el nowcasting invita a tomarlas de inmediato (OMM, 2017).

\section{SOCIEDADES PREPARADAS PARA EL NOWCASTING}

Tomar decisiones y hacer útil la predicción meteorológica en el rango del nowcasting exige sociedades avanzadas. Esta predicción genera un continuo flujo de información, cada pocos minutos, y con elevada resolución espacial, que debe ser gestionada óptimamente por el binomio suministrador-usuario.

Tradicionalmente, los usuarios del nowcasting han recibido mensajes, llamadas telefónicas o avisos inminentes, referidos a fenómenos adversos. Sin embargo, están cada vez más preparados y disponen de más recursos, y demandan del nowcasting más variables asociadas a tiempo adverso, y con más detalle local y temporal. Además, requieren avisos más precisos, claros y difundidos por más medios. Por otra parte, la demanda de nowcasting incluye productos precisos y puntuales de tiempo no adverso, tales como observaciones y elementos meteorológicos de alta resolución, para ser ingestados en sus propias aplicaciones, referidas en inglés como downstream applications (OMM, 2017).

Respecto al uso que los usuarios finales están haciendo de los productos de nowcasting, estamos presenciando un cambio de paradigma, ya que han empezado a tomar sus propias decisiones con tan solo echar un vistazo a sus aplicaciones móviles, principalmente basadas en la precipitación estimada por el radar.

En la predicción meteorológica, cada rango temporal requiere una herramienta diferente, lo que implica diversas resoluciones, formatos, umbrales, intervalos, etc. Con frecuencia, el usuario encuentra problemas en su interpretación. La predicción del tiempo en los primeros minutos está basada en la observación y su extrapolación, así como en técnicas estadísticas o heurísticas. La capacidad predictiva de estas técnicas varía desde minutos a horas, dependiendo del fenómeno. Más allá de las primeras horas, la mejor solución la ofrecen los modelos numéricos de predicción del tiempo (NPT). Sin embargo, los usuarios están exigiendo productos denominados «sin costuras», en inglés seamless, que mostrando las mismas variables y formatos proporcionen, en cada momento de la predicción, la herramienta que más pericia tenga. Atender a estos usuarios exige ensamblar adecuadamente las soluciones de las distintas herramientas.

El nowcasting, como todas las predicciones, debe ser calibrado. A diferencia de otros rangos, los usuarios de la predicción inmediata requieren métricas de calibración adaptadas a sus intereses, de forma que el producto optimice sus decisiones y no tanto unos neutros indicadores estadísticos. Para llevar a cabo esta tarea, es necesaria una fluida comunicación entre el suministrador y el usuario (OMM, 2017).

\section{AVANCES EN LOS SISTEMAS DE NOWCASTING}

En paralelo a la mayor preparación y recursos de los usuarios del nowcasting, la predicción en las primeras horas también está mejorando. Estos avances están pasando por disponer de más observaciones y mejor adaptadas, lo que facilita la elaboración de análisis más detallados, en ocasiones de carácter probabilístico, y de nuevas técnicas de nowcasting basadas en ensembles, o conjunto de predicciones (WANG, 2017). 
Respecto a la mejora en los sistemas de observación desde superficie, son de especial relevancia las nuevas redes de estaciones meteorológicas automáticas que, combinadas, comienzan a ofrecer detalles de estructuras espaciotemporales propias del nowcasting. Un caso extremo, por su alta densidad de observaciones, es el sistema conocido con sus siglas en inglés $L L W A S$, cuya red de anemocinemómetros en torno a aeropuertos detecta automáticamente cizalladura del viento junto al suelo. Por otro lado, las redes de detección de descargas eléctricas están impulsando nuevas técnicas de nowcasting orientadas a objetos convectivos, mejorando su identificación, seguimiento y extrapolación. Además, dan pistas de posible severidad asociada a cambios en la frecuencia y altura de las descargas, modelo conceptual conocido en inglés como lightning jump. La tecnología está facilitando el desarrollo de sensores automáticos de nuevas variables meteorológicas, como el tipo de precipitación. En un futuro próximo, a la mejora del nowcasting se incorporarán observaciones no convencionales, como las realizadas desde vehículos, dispositivos móviles, etc. (OMM, 2017).

La observación de variables de altura está siendo clave para satisfacer las necesidades de usuarios. En algunos aeropuertos, los radares Doppler, de siglas en inglés $T D W R$, o los perfiladores de viento, informan en tiempo casi real a los controladores de tráfico aéreo sobre posible cizalladura del viento en altura. Las observaciones desde aviones, $A M D A R$ en sus siglas en inglés, son útiles para identificar áreas de turbulencia, ajustar la isocero y el tipo de precipitación esperada, la profundidad de una inversión, etc. (COMET/UCAR, 2014).

Desde los satélites, la observación está posibilitando el análisis del tipo de nubes, de la precipitación asociada, o de los vientos que, además, facilitan la extrapolación de algunos de estos campos. Las imágenes permiten la identificación de los objetos convectivos en rápido desarrollo, o valorar la inestabilidad del aire (NWCSAF, 2018). La nueva generación de hidroestimadores resulta prometedora, combinando la alta frecuencia de observación de las plataformas geoestacionarias, con la información del interior de las nubes aportada por los fotómetros en microondas desde las plataformas polares. Las técnicas RGB de visionado de imágenes están facilitando la identificación de áreas nubosas reveladoras de posible severidad. Los futuros satélites, como el MTG, incorporarán un detector de descargas, el instrumento LI, lo que sin duda mejorará los sistemas de nowcasting (EUMETSAT).

El radar meteorológico es en muchos casos la herramienta más útil para el nowcasting de la precipitación y fenómenos asociados. Los esfuerzos por aumentar la frecuencia de exploración cada 5 o menos minutos, su capacidad Doppler, y la doble polarización, están mejorando el análisis de hidrometeoros. Además, los avances en las técnicas de extrapolación, tanto de objetos convectivos como del campo radar, intentan reducir la incertidumbre del nowcasting, tratando incluso de estimarla. Los productos de acumulación de precipitación basados en observaciones y en extrapolaciones radar son básicos en los nuevos sistemas de alerta temprana de inundaciones.

Además de la observación, los modelos NPT están reforzando su apoyo al nowcasting. Tradicionalmente, la contribución de estos ha sido mayor en la predicción a muy corto plazo, y enfocado a eventos de escala mesoalfa o mayores. Las exigencias temporales y espaciales de la predicción en este rango necesitan modelos con ciclos de actualización al menos horarios, teniendo que ejecutarse en periodos muy cortos de tiempo. Como ejemplo, MétéoFrance desarrolla el Arome-Nowcasting, un modelo que asimila la información radar cada hora, mientras que para el resto de variables lo hace con menor frecuencia, con un tiempo de spin-up de unos 20 minutos, y dejando disponible la predicción en unos 30 minutos (Ludovic et al., 2015).

Además de los ciclos horarios, son varios los esfuerzos en la mejora de los modelos NPT para el nowcasting, de los que cabe destacar: la asimilación de datos y el análisis, el uso de modelos de convección permitida, y el ensamblaje entre estos con el nowcasting. El futuro apunta a productos que ensamblen no solo una, sino múltiples extrapolaciones con sistemas de predicción por conjuntos basados en modelos de convección permitida (WANG, 2017). 


\section{NOWCASTING EN LOS SERVICIOS METEOROLÓGICOS DE NUESTRO ENTORNO}

Los sistemas de nowcasting desarrollados recientemente en los Servicios Meteorológicos Nacionales (SMN), de nuestro entorno aumentan su componente de automatismo, integrando distintos tipos de datos y observaciones. La pronta caducidad de la información proporcionada por el nowcasting hace tender a técnicas pragmáticas en detrimento de la resolución más en profundidad del comportamiento de los sistemas meteorológicos. En nowcasting, lo importante es ofrecer soluciones con rapidez, aunque sean aproximadas, por medio de métodos en ocasiones heurísticos. Estos sistemas proponen avisos, emiten mensajes y llamadas de atención al predictor, quien debe valorar su conveniencia.

La predicción en el rango del nowcasting ha sido resuelta con estrategias muy diversas. Clasificar los sistemas y técnicas de nowcasting conlleva cierta ambigüedad. En este artículo se propone la sugerida por la OMM (OMM, 2017), que diferencia los sistemas de nowcasting en:

1. Los que identifican, siguen y extrapolan objetos convectivos.

2. Los que realizan seguimiento y extrapolación de campos.

3. Los que combinan múltiples observaciones.

4. Las aproximaciones probabilísticas.

Bajo esta clasificación, se muestran algunos interesantes ejemplos de sistemas de nowcasting operativos en SMN de nuestro entorno.

\subsection{Sistemas de identificación, seguimiento y extrapolación de objetos convectivos}

El SMN alemán, DWD, utiliza el productos CellMOS, basado en técnicas estadísticas conocidas en inglés como Model Output Statistic (MOS), para predecir ciertas características de las células convectivas (DWD). La técnica consiste en calcular los coeficientes de una ecuación de regresión múltiple en base a una serie larga de pares de datos previstos por el modelo NPT y observadas. Conocidos los coeficientes MOS, y a partir de las predicciones del modelo y de las observaciones, se infiere el valor de las variables a predecir. De esta

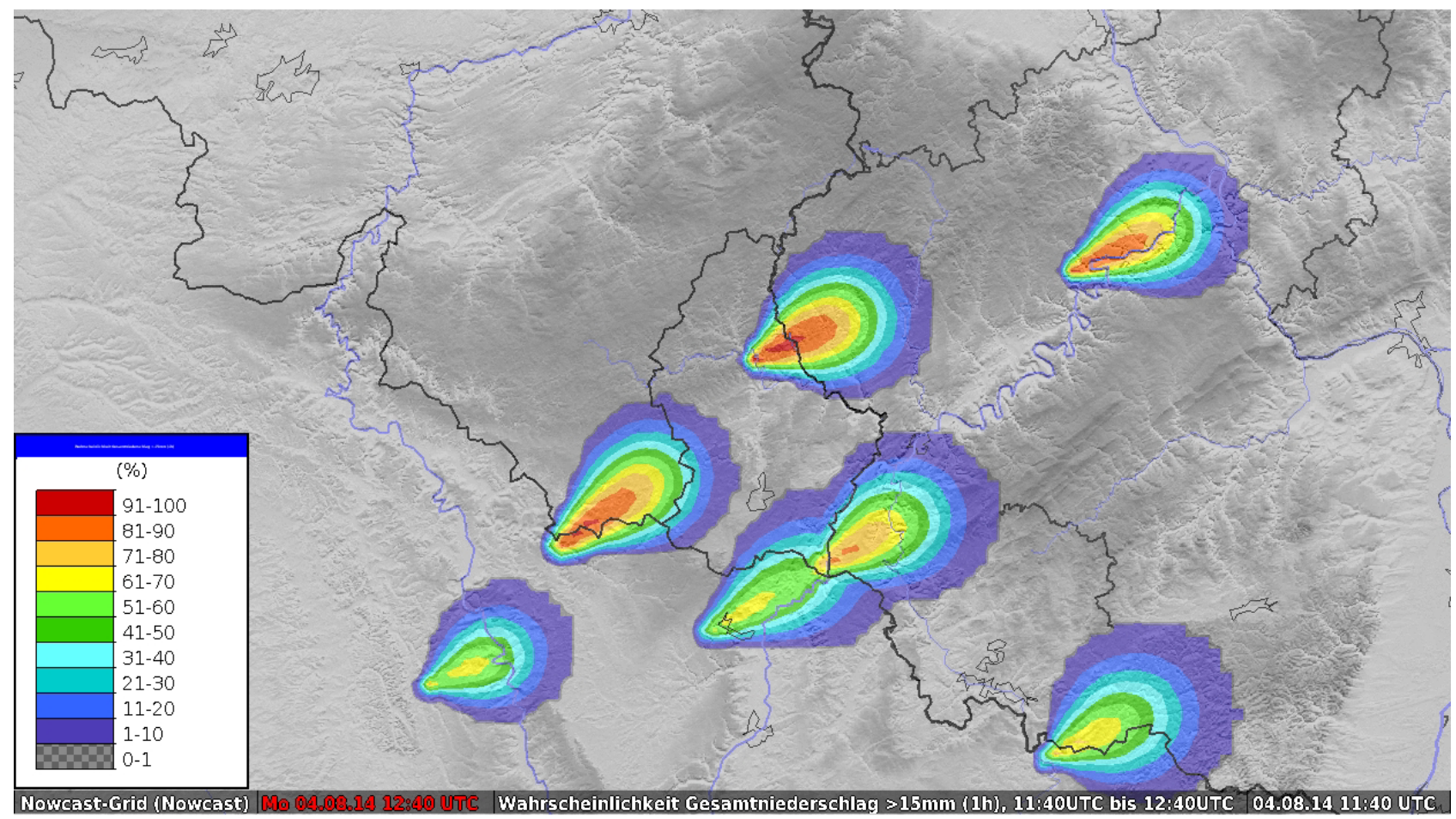

Figura 1. Probabilidad de precipitación mayor de $15 \mathrm{~mm} / \mathrm{h}$ para varios objetos convectivos, mostrado por el producto CellMOS del DWD. Elaboración del DWD. 
forma, con los datos del modelo, y de las observaciones de radar y rayos, se prevén características del objeto convectivo: presencia de granizo, precipitaciones sobre ciertos umbrales, etc.

MétéoFrance dispone del producto SIGOONS (BROvELLI, 2005) que, además de en objetos convectivos, enfoca su atención en otros objetos como áreas de niebla, zonas con viento fuerte, etc. Es un sistema híbrido hombre-máquina ya que, aunque está diseñado para funcionar de forma automática, el predictor arbitra las discrepancias entre los objetos extrapolados y las observaciones, pudiendo, además, seleccionar zonas propensas a la convección.

\subsection{Sistemas para el seguimiento y extrapolación de campos}

El sistema 2PiR de MétéoFrance realiza extrapolación considerada semilagrangiana, dividiendo el campo radar y calculando un vector de desplazamiento para cada parcela. El producto ofrece, para localidades francesas, respuesta a la pregunta ¿va a llover en la próxima hora en su localidad?, indicando, en intervalos de 5 minutos, si esta va a ser débil, moderada o fuerte, e informando hasta qué punto el producto es válido en función del lugar y la posición del radar.

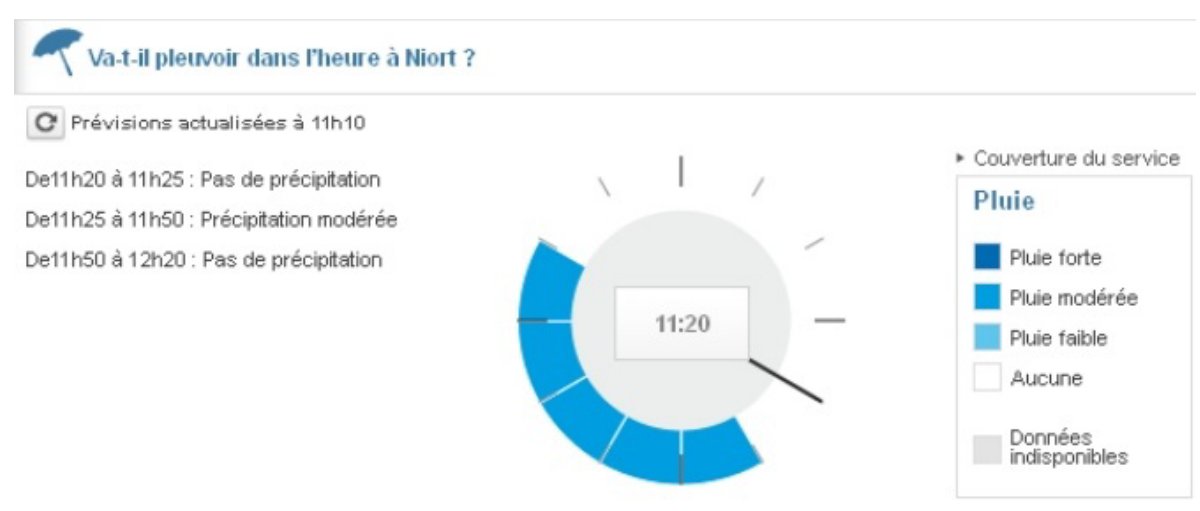

Figura 2. ¿Va a llover en la próxima hora en tu localidad? Previsión de lluvias del producto 2 PiR de MétéoFrance en intervalos de 5 minutos, con indicación de la intensidad. Elaboración de MétéoFrance.

De similares características al anterior es el producto del DWD RadVor-Op que extrapola, hasta 2 horas en intervalos de 5 minutos, el campo radar (DWD). Este producto ha sido desarrollado para optimizar la gestión del riesgo de inundaciones, y entre otros resultados, ofrece el acumulado de precipitación prevista en la siguiente hora, y analiza y prevé la presencia de granizo y de variables típicas de invierno.

Los productos de extrapolación de campos no se limitan a los de radar, ya que los hay que extrapolan la nubosidad detectada con satélites geoestacionarios, como EXIM del NWCSAF. Un buen ejemplo de aplicación

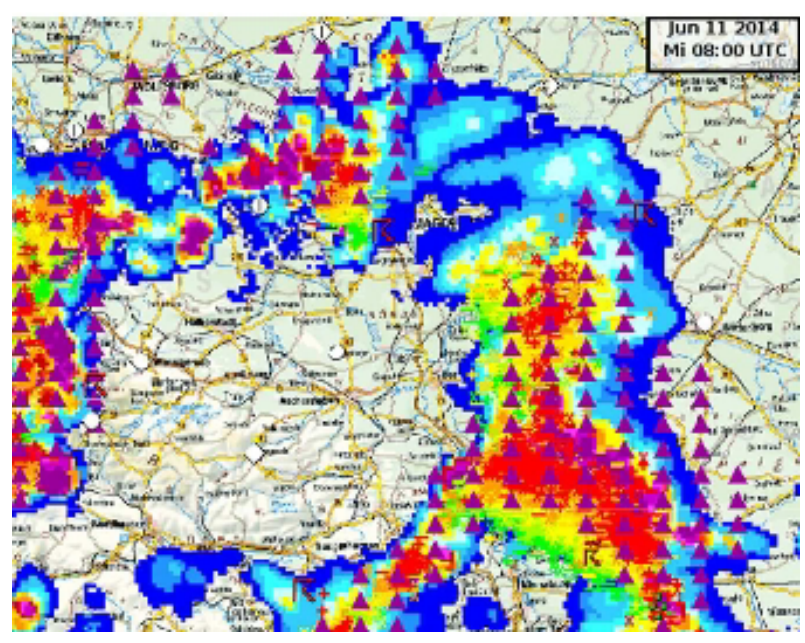
de estos sistemas es el NowcRadiation de AEMET, del que se infiere la radiación que llega a puntos determinados a breves intervalos de tiempo. El producto ensambla en el periodo de 1 a 4 horas de predicción, la nubosidad extrapolada con la de un modelo NPT de convección permitida.

Figura 3.

Producto de extrapolación del campo radar RadVor-Op del DWD, con indicación empírica de la presencia de granizo. Elaboración del DWD. 


\subsection{Sistemas que combinan múltiples observaciones}

La combinación óptima de las observaciones de diferentes fuentes para la práctica del nowcasting es todo un reto. Enfocado a los sistemas convectivos, el sistema NowcastMIX del DWD proyecta en una rejilla de 1 por $1 \mathrm{~km}$ los objetos convectivos identificados en otros productos, los datos de rayos, el análisis y las extrapolaciones radar, los datos de superficie, y de los modelos NPT. Tras aplicar técnicas de lógica difusa establece la categoría de los avisos asociados a la convección (lluvia, granizo o rachas), y tras aplicar filtros espaciales de suavizado, emite avisos cada 5 minutos de eventos inminentes.

El sistema INCA, Integrated Nowcasting Comprehensive Analysis, del ZAMG, implantado en varios $\mathrm{SMN}$ europeos, extiende el nowcasting más allá de la precipitación y objetos convectivos, al extrapolar durante las primeras dos horas, además del campo radar, la nubosidad observada por satélite. El producto realiza análisis en alta resolución de tem-

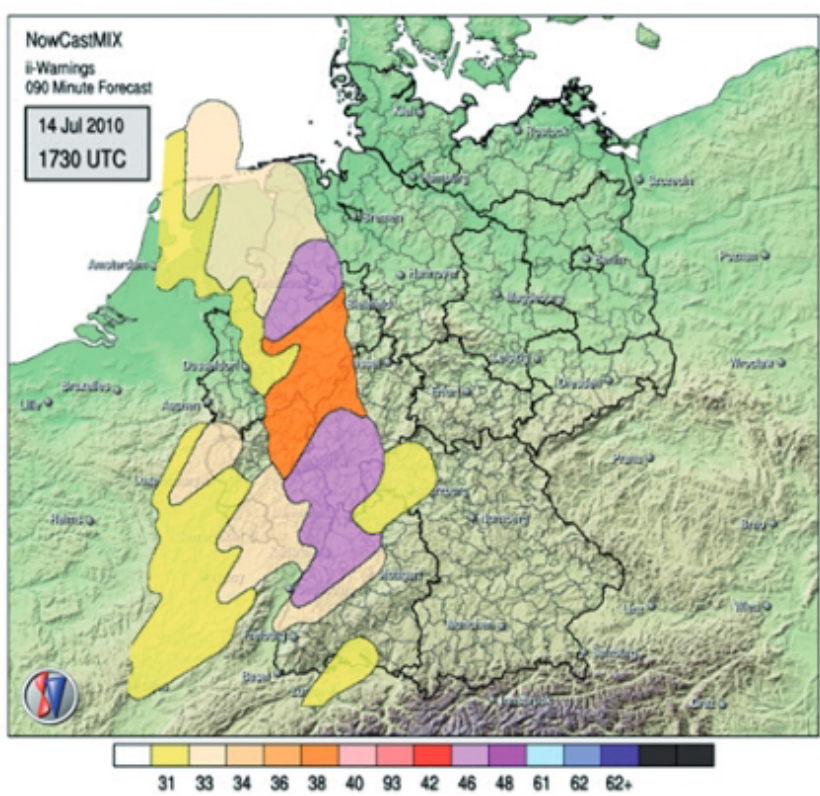

Figura 4. Avisos propuestos (numeración de la escala) por el producto NowCastMIX para los próximos 90 minutos. Elaboración del DWD. peratura, humedad y viento, que evolucionan con la tendencia indicada por el modelo NPT. A partir de las seis horas, la predicción está resuelta completamente por el modelo, y, entre las dos y seis horas, la predicción es una combinación entre las extrapolaciones y el modelo.

\subsection{Aproximaciones probabilísticas}

Una tendencia de los sistemas de nowcasting es la de ofrecer información de la incertidumbre en todos los rangos de predicción. El producto STEPS (Short Term Ensemble Prediction System) de MetOffice, separa el campo de precipitación radar en diferentes tamaños, de manera que las mayores estructuras de precipitación, que suelen ser más predecibles, pueden extrapolarse más tiempo, mientras que los eventos pequeños, menos. Más allá de estos límites de predecibilidad, el producto combina esta información con la de un modelo NPT para las estructuras mayores de precipitación, usando métodos estocásticos para reproducir las formas más pequeñas. El término ensemble del producto se refiere al hecho de que se producen muchas predicciones, generando vectores de desplazamiento de la lluvia ligeramente diferentes unos de otros, o generando formas pequeñas de lluvia mediante estadísticas aleatorias. Con aproximaciones de este tipo, se puede estimar la incertidumbre asociada a la predicción de acumulaciones importantes de lluvia (MetOffice).

Figura 5.

Diferentes extrapolaciones de la precipitación radar generadas por el producto STEPS del MetOffice.

Elaboración del MetOffice.
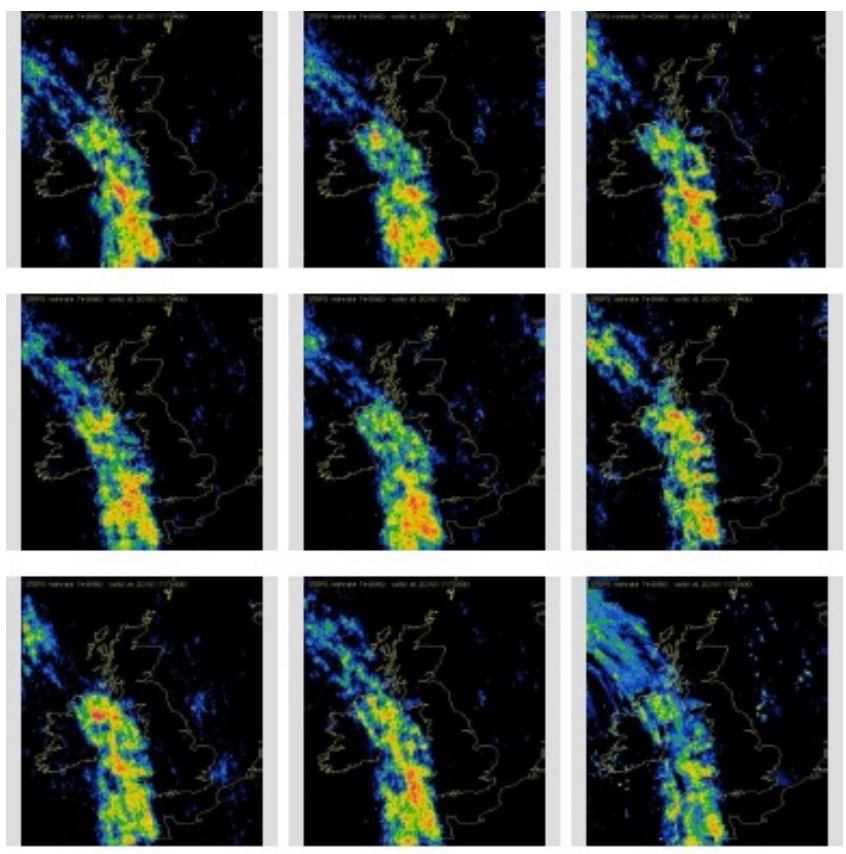

Control 
El producto RAVAKE finlandés del FMI es un sistema probabilístico de la predicción de precipitación, que elabora 51 extrapolaciones del campo radar, así como 51 miembros de un sistema por conjuntos de hombre pobre de un modelo NPT de alta resolución, y 51 miembros de un modelo NPT por conjuntos. El producto principal del sistema consiste en la predicción, a resolución de 1 km, de la precipitación y de la probabilidad de que esta exceda diferentes umbrales, en un intervalo temporal que va desde los 15 minutos a los 5 días. Esta continuidad sin costuras se consigue ensamblando (técnica conocida en inglés como blending) los tres conjuntos de ensembles en el dominio temporal (HEINONEN et al., 2013).
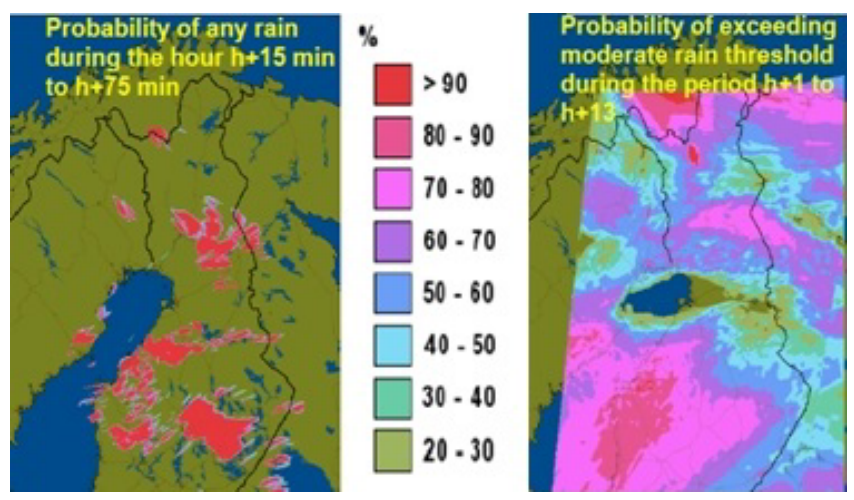

Figura 6. Probabilidad de lluvia entre los próximos 15 y 75 minutos (izquierda), y de que esta supere el umbral de moderada entre la próxima hora y las 12 siguientes (derecha), según el producto RAVAKE del FMI. Elaboración del FMI.

\section{EL PREDICTOR DE NOWCASTING}

La práctica del nowcasting con tiempo adverso puede resultar desbordante. El predictor del tiempo en este rango, referido en algunos textos como nowcaster, debe comenzar su tarea acotando los fenómenos esperables, debe analizar cada nueva observación, y practicar un diagnóstico continuado del tiempo, elaborando un modelo mental en cuatro dimensiones del estado de la atmósfera y de las estructuras de mesoescala que en ella se desarrollan.

Dado lo ocasional de las situaciones de tiempo adverso, la tabla de tareas del nowcaster suele llenarse con actividades distintas a la vigilancia. Llegado el tiempo severo, el predictor debe priorizar la vigilancia en detrimento de las tareas rutinarias. La práctica del nowcasting requiere tiempo de calidad, dedicación exclusiva, elevado nivel de concentración, y todo el apoyo disponible, incluida la automatización de las tareas rutinarias. Cualquier esfuerzo en adaptar los productos y herramientas para su rápida interpretación ayudará al predictor a tomar decisiones rápidas.

Una pieza clave en este rango de predicción es la integración de las observaciones de distintas fuentes que, de manera casi inmediata, ofrezcan al predictor su fácil lectura. Suele ser aconsejable disponer de guías rápidas para la interpretación de los productos: umbrales a vigilar, dependencia de la época del año, limitaciones en su uso, etc. Igual de útil pueden resultar las guías rápidas de ciertos modelos conceptuales que recuerden al predictor las estructuras que debe identificar en cada sistema de observación. Por último, resultan especialmente aconsejables los sistemas de emisión automática de mensajes, que llaman la atención al nowcaster y le ayudan en sus tareas, siendo estas técnicas más necesarias cuanto mayor sea el área de vigilancia.

\section{CONCLUSIONES}

El artículo subraya las diferencias entre el nowcasting y la predicción a muy corto plazo, a pesar del solape en sus rangos de predicción. Destaca el aumento de requisitos y la mayor exigencia que tienen los actuales usuarios del nowcasting. Orienta las causas en las mejoras de la predicción inmediata, principalmente basadas en más observaciones y más adaptadas, en mejores técnicas de extrapolación, y mejores modelos NPT. Plantea una clara apuesta por soluciones probabilísticas y sin costuras en los sistemas de nowcasting. Se muestran ejemplos de productos operativos en diferentes SMN de nuestro entorno: orientados a objetos convectivos, a la extrapolación de campos, sistemas que realizan nowcasting de múltiples variables, y aproximaciones probabilísticas. Finalmente, se plantean algunas de las necesidades del nowcaster. 
La principal conclusión es que, satisfacer las necesidades de los usuarios del nowcasting exige sistemas con más pericia, ensamblados y con información de su incertidumbre en tiempo real. Estos sistemas, cuando sea pertinente, deberán suministrar la información directamente al usuario. El nowcaster podrá aportar valor en muchas situaciones, pero a costa de alta preparación y dedicación.

A diferencia de otros rangos de predicción, el nowcasting está íntimamente ligado al uso que se haga de sus productos, lo que la convierte en una actividad multidisciplinar y estrechamente ligada a sus usuarios.

\section{AGRADECIMIENTOS}

Gran parte de este trabajo es fruto de las ideas y esfuerzos previos de otras personas. En este caso, mi agradecimiento al equipo de expertos del proyecto ASIST de EUMETNET, en especial, a la experiencia vertida por Yong Wang en diversos documentos, y a Paolo Ambrosetti por compartir sus conocimientos. Agradecer también al personal de la Delegación Territorial de AEMET en Murcia, con quien he discutido alguno de los temas tratados. Y gracias a Alicia por sus valiosas revisiones.

\section{REFERENCIAS}

AMBRosetTi et al., 2014. Statement of Guidance for Nowcasting and VSRF.

BrownING, K. A., 1992. Nowcasting.

BROVELli et al., 2005. SIGnificant weather Object Oriented Nowcasting System.

COMET/UCAR, 2014. Introduction to AMDAR.

DWD. www.dwd.de.

EUMETSAT. www.eumetsat.int.

HEINONEN et al., 2013. Improved wet weather wastewater influent modelling at Viikinmäki WWTP by on line weather radar information.

INM, 198?. Vigilancia y VSRF. Consideraciones generales y estado actual en el INM.

LUDOVIC et al., 2015. AROME-NWC: a new nowcasting tool based on an operational mesoscale forecasting system.

MétéoFrance. www.meteofrance.com.

MetOffice. www.metoffice.gov.uk.

NWCSAF. www.nwcsaf.org.

Orlansky, I., 1975. A Rational Subdivision of Scales for Atmospheric Processes.

WANG, Y., 2017. Nowcasting Forecasting Activities (presentation in the Nowcasting and Mesoscale Working Group Meeting, Buenos Aires, Argentina).

World Meteorological Organization, 2017. Guidelines for Nowcasting Techniques, WMO-No. 1198.

ZAMG. www.zamg.ac.at. 